\title{
A Probabilistic Estimation of Earthquake Occurrence on the Basis of the Appearance Times of Multiple Precursory Phenomena
}

\author{
Kenji Maeda* and Akio Yoshida \\ Meteorological Research Institute, Tsukuba 305, Japan
}

\begin{abstract}
Appearance times of earthquake precursory phenomena are analyzed to calculate the synthesized probability of earthquake occurrence when multiple disciplines of precursors are observed. Various disciplines of precursors are classified into three groups: long, middle and short terms. We assume they are independent mutually. By analyzing the precursor time $\left(T_{\mathrm{p}}\right)$ data for each term using Weibull distribution function, it is found that distributions of $T_{\mathrm{p}}$ data for long, middle, and short terms have the properties of increasing, decreasing, and nearly constant hazard rates, respectively. Using Weibull parameters estimated for each term, we calculate the conditional probability of earthquake occurrence on the basis of the appearance times of precursors; where "conditional" means calculating probability on the condition that no earthquake has happened yet by the current time. Reliability parameter $r$ is introduced for each term to take account of such a possibility that a part of the "precursors" are spurious ones. Introducing of the parameter $r(<1)$ has an effect to makke the conditional probability for every term decrease monotonously long after the appearance of each precursor. The synthesized probability is obtained by the formula of Utsu $(1977,1983)$. A probability change is calculated for the case of the Izu-Oshima-Kinkai earthquake of 1978 by applying this method to the observed precursor data.
\end{abstract}

\section{Introduction}

It is expected that many kinds of geophysical or geochemical anomalies will be observed before a large earthquake. In fact, many kinds of anomalies were observed before the 1975 Haicheng earthquake $(M=7.3)$ (Rikitake, 1982), the 1976 Tangshan earthquake $(M=7.8)$ (Oike, 1978; Rikitake, 1982; Yong et al., 1988), the 1923 Kanto earthquake $(M=7.9)$ (Rikitake, 1986), the 1944 Tonankai earthquake $(M=7.9)$ (Rikitake, 1986; E.C.S.S., 1986), and the 1978 Izu-Oshima-Kinkai earthquake $(M=7.0)$ (Rikitake, 1982, 1986). However, we have found so far no precursory phenomenon whose physical mechanism is clarified and which can be used to predict occurrence of earthquakes deterministically. Consequently, a probabilistic approach using various kinds of precursory phenomena is considered to be one of the practical approaches for earthquake prediction.

Received September 14, 1990; Accepted February 4, 1991

* To whom correspondence should be addressed. 
It would be reasonable in a probabilistic sense to conclude that if several kinds of anomalies are observed at once and those anomalies can be treated as independent, the probability of occurrence is higher than in the case that a single anomaly is observed. Utsu $(1977,1983)$ formulated a method of calculating the probability for such cases that multi-element precursors are observed, and Utsu $(1979,1983)$ and Rikitake (1987 b) applied the method to the Izu-Oshima-Kinkai earthquake of 1978. Aki (1981) derived the same formula of probability for multiple independent precursors by using the Bayes' theorem.

On the other hand, there are some models for calculating time-dependent probability based on a single precursor. Investigating the precursor time distribution in short time range of 0.1 to 40 days, Hamada $(1987,1988)$ introduced a power-law type function. Recurrence time distributions of large earthquakes would also provide us with probability functions for the occurrence of a next earthquake. In order to analyze the recurrence time distributions, normal (Sykes and Nishenko, 1984), lognormal (Vere-Jones, 1978; Nishenko and Buland, 1987) or Weibull (Hagiwara, 1974; Rikitake, 1976) distributions, etc., have been proposed.

In this paper, we calculate the time-dependent probability in the case that multiple independent precursors which may contain spurious ones are observed, applying the Weibull distribution analysis to the appearance time data of precursory phenomena.

\section{Data}

Several kinds of compiled data about precursory phenomena are published at present (e.g., Niazi, 1985; E.C.S.S., 1985; Rikitake, 1986; Hamada, 1987). Recently we have also compiled (S.V.R.D., 1990) precursory phenomena in the world that had been reported in easily accessible documents. About 1,100 precursor data for about 530 earthquakes have been compiled. The numbers of data belonging to each discipline are listed in Table 1. More than half are seismic precursors and no such data of anomalies as animal behavior, plant, climate, lights and so on are included.

We calculate the probability of earthquake occurrence on the basis of the distributions of the precursor time $T_{\mathrm{p}} . T_{\mathrm{p}}$ is defined as the time interval between appearance of a precursory phenomenon and occurrence of an earthquake. It is well known for some kinds of precursors that logarithm of $T_{\mathrm{p}}$ is linearly related with the main shock magnitude (e.g., Scholz et al., 1973; Rikitake, 1987 a). To take the dependence on magnitude into consideration, it is desirable that the probability is calculated for each magnitude range. On the other hand, it is required that the number of data is large enough to obtain statistically meaningful results. Therefore we use the data only for large earthquakes with magnitude equal to or larger than 6.5. As a result, the number of $T_{\mathrm{p}}$ data used in this study amounts to 357 in total.

\section{Methods}

First, we classified disciplines into three groups: long, middle and short terms. We assume that disciplines whose ranges of $T_{\mathrm{p}}$ differ much from each other are independent mutually. This assumption may not be justified when precursors belonging to different 
Table 1. Number of data for each discipline which are compiled by S.V.R.D. (1990).

\begin{tabular}{|c|c|c|c|}
\hline Discipline & $\begin{array}{l}\text { Number } \\
\text { of data }\end{array}$ & Discipline & $\begin{array}{l}\text { Number } \\
\text { of data }\end{array}$ \\
\hline Gravity & 3 & Foreshock activity & 228 \\
\hline Geodetic survey & 24 & $b$-value & 16 \\
\hline Tide-gauge observation & 27 & $Q$-value & 8 \\
\hline Other geodetic precursors & 3 & Focal mechanism & 33 \\
\hline \multirow[t]{2}{*}{ Total of geodetic precursors } & 57 & $\begin{array}{l}\text { Seismic wave velocity } \\
\text { Seismic activity pattern }\end{array}$ & $\begin{array}{l}12 \\
25\end{array}$ \\
\hline & & Seismic pre-activity & 72 \\
\hline Tilt (horizontal pendulum) & 43 & Seismic gap or quiescence & 119 \\
\hline Tilt (water-tube) & 11 & Seismic wave form & 16 \\
\hline Tilt (bore-hole) & 6 & Seismic wave spectrum & 17 \\
\hline Tilt (others) & 15 & & \\
\hline Extensometer & 45 & Total of seismic precursors & 546 \\
\hline Volumetric strainmeter & 21 & & \\
\hline Crustal stress & 45 & Geomagnetic field & 19 \\
\hline Acoustic emission & 3 & Earth current & 56 \\
\hline \multirow[t]{7}{*}{ Total of tilt, strain, stress } & 189 & $\begin{array}{l}\text { Resistivity } \\
\text { Resistivity (Yamazaki-meter) }\end{array}$ & $\begin{array}{l}17 \\
34\end{array}$ \\
\hline & & Electromagnetic radiation & 16 \\
\hline & & Total of electromagnetic precursors & 142 \\
\hline & & Radon & 63 \\
\hline & & Component of groundwater, gas & 55 \\
\hline & & Underground water-level, hot spring & 47 \\
\hline & & Total of geochemical precursors & 165 \\
\hline & & Total & 1,099 \\
\hline
\end{tabular}

terms are originated from the same cause. The effect of dependence will be discussed briefly. In order to classify disciplines into three terms, we adopted a criterion that more than half of $T_{\mathrm{p}}$ data in each discipline satisfy the following condition: $T_{\mathrm{p}} \geqq 1,000$ days for long term disciplines; 10 days $\leqq T_{\mathrm{p}} \leqq 1,000$ days for middle term disciplines; $T_{\mathrm{p}} \leqq 10$ days for short term disciplines. According to this criterion, we can classify three disciplines of geodetic survey, seismic pre-activity, and seismic quiescence as long term precursors, six disciplines of tilt, crustal stress, earth current, resistivity, radon, and chemical components in groundwater or gas as middle term precursors, and one discipline of foreshock activity as short term precursor. Precursors observed by the Yamazaki-meter are excluded from the data of resistivity because the distribution pattern of $T_{\mathrm{p}}$ is much different from that of the other type of resistivity and the data may be valid only at a special observation point (Aburatsubo). The rest of the disciplines in Table 1 cannot be classified into any term because they do not satisfy the criterion or the numbers of data are too small to be treated statistically. In Fig. 1, we show the distributions of logarithm of $T_{\mathbf{p}}$ measured in days for the three terms in the form of 
histograms and cumulative curves.

Next, on the basis of these three types of distribution data, we introduce a simple model to approximate them. Hamada $(1987,1988)$ obtained a power-law type function for the short-term $T_{\mathrm{p}}$ distribution, analyzing precursors whose $T_{\mathrm{p}}$ are in the range of 0.1 to 40 days. On the other hand, normal (Sykes and Nishenko, 1984), lognormal (Nishenko and Buland, 1987), Weibull (Hagiwara, 1974; Rikitake, 1976) distributions or more complex type (e.g., Ogata and Katsura, 1986) of distribution functions are proposed to be fitted to empirical distributions of earthquake recurrence time. In the present paper, Weibull function is used to approximate the distribution of $T_{\mathrm{p}}$ data for each term on the analogy of industrial failure time statistics and for the simplicity of expression. Another reason for using Weibull function is that the character of distributions, e.g., increasing, decreasing, or constant hazard rates, can be easily indicated by one parameter. Following the manner of Rikitake (1976), the probability density function $f(t)$ of Weibull distribution can be put as

$$
f(t)=K t^{m} \exp \left[-K t^{m+1} /(m+1)\right],
$$

where $t=T_{\mathrm{p}}$ in our case and $K$ and $m$ are parameters which will be determined to fit the distribution. The cumulative probability $F(t)$, which is the probability of earthquake occurrence during the period from the appearance of a precursor (defined as $t=0$ ) through the current time $t$, is obtained as

$$
F(t)=\int_{0}^{t} f(t) \mathrm{d} t=1-\exp \left[-K t^{m+1} /(m+1)\right]
$$

and the probability $R(t)$ that no earthquake occurs during that period becomes

$$
R(t)=1-F(t)=\exp \left[-K t^{m+1} /(m+1)\right] .
$$

The conditional probability density (hazard rate) $\mu(t)$ under the condition that no earthquake has occurred till current time $t$ is defined as the ratio of $f(t)$ to $R(t)$, which leads to

$$
\mu(t)=\frac{f(t)}{R(t)}=K t^{m},
$$

using Eqs. (1) and (3). We can easily understand from Eqs. (1) and (4) that each case of $m<0, m=0$, and $m>0$ corresponds to decreasing, constant, and increasing distribution of hazard, respectively. If we take the double logarithm of $1 / R(t)$, Eq. (3) comes to

$$
\ln \{\ln (1 / R(t))\}=K^{\prime}+(m+1) \ln (t),
$$

where $K^{\prime}=\ln \{K /(m+1)\}$. This shows the linearity between the double logarithm of $1 / R(t)$ and logarithm of $t$. For actually observed $T_{\mathrm{p}}$ data, $R(t)$ can be defined as

$$
R\left(t_{i}\right)=1-\sum_{j=0}^{i-1}\left(n_{j} / N\right), \quad t_{i}=\sum_{j=0}^{i-1} \Delta t_{j}, \quad(i, j=0,1,2, \cdots)
$$

where $\Delta t_{i}$ is an $i$-th time interval suitably chosen, $n_{j}$ is the number of $T_{\mathrm{p}}$ data falling in 

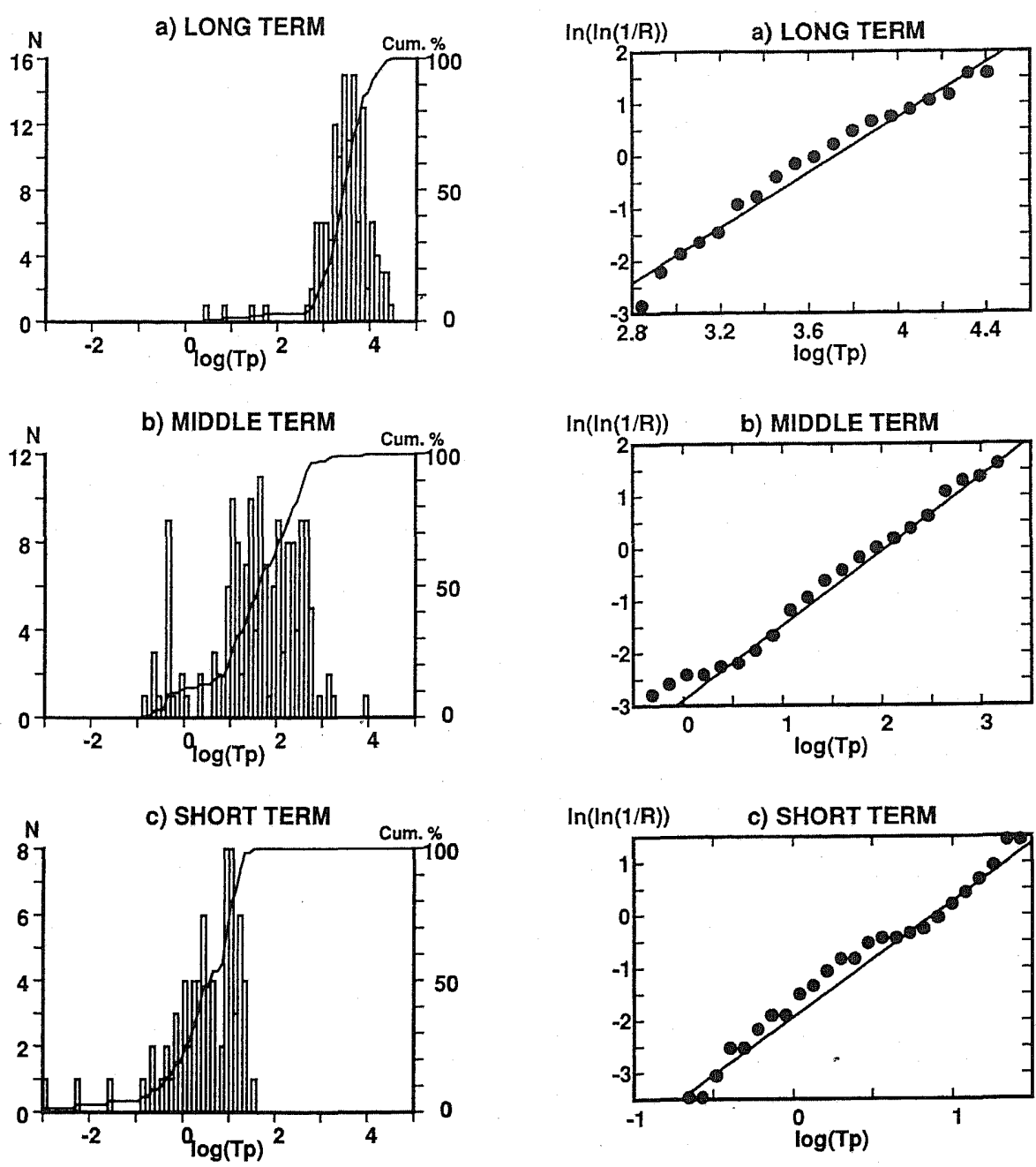

Fig. 1

Fig. 2

Fig. 1. Histograms and cumulative curves of precursor time $\left(T_{\mathrm{p}}\right)$ data for each term. $T_{\mathrm{p}}$ is measured in days.

Fig. 2. Variation of $\ln \left\{\ln \left(1 / R\left(t_{i}\right)\right)\right\}$ as a function of $\log _{10}\left(T_{\mathrm{p}}\right)$ and calculated line are shown for each term. $R\left(t_{i}\right)$ is defined by Eq. (6) (see the text) and $T_{\mathrm{p}}$ is measured in days. Calculated curves are obtained from Eq. (5) (see the text) by applying the values of $K$ and $m$ in Table 2 .

the time range between $t_{j}$ and $t_{j+1}$ and $N$ is the total number of the data. To determine the parameters $K$ and $m$ from actual data, Rikitake (1976) conveniently applied the least squares method to Eq. (5) and Utsu (1984) used the method of moments or maximum likelihood. We adopted the values of parameters estimated by the maximum

Vol. 38 , No. 6, 1990 
Table 2. Estimated values of parameters $K, m$, and total numbers of used $(N)$ and original $\left(N_{0}\right)$ data for each term.

\begin{tabular}{llrrr}
\hline Term & \multicolumn{1}{c}{$K$} & $m$ & $N$ & $N_{0}$ \\
\hline Long & $6.58 \mathrm{E}-5$ & 0.139 & 125 & 131 \\
Middle & 0.0341 & -0.381 & 151 & 157 \\
Short & 0.138 & -0.0439 & 65 & 69 \\
\hline
\end{tabular}

likelihood method, which are not much different from ones estimated by Rikitake's method.

The data of $\ln \left\{\ln \left(1 / R\left(t_{i}\right)\right)\right\}$ and the calculated lines for each term of precursors are shown in Fig. 2. Note that $T_{\mathrm{p}}$ is measured in days and expressed as $\log _{10}\left(T_{\mathrm{p}}\right)$ in Fig. 2. The estimated values of $K$ and $m$, the total number of data used to estimate the values, $N$, and total number of original data, $N_{0}$, for each term are listed in Table 2. It should be noted that the estimated values are obtained by excluding some data which deviate much from the lines. The excluded data drop in the range that $T_{\mathrm{p}}<600$ days for long term, $T_{\mathrm{p}}<0.32$ days or $T_{\mathrm{p}} \geqq 2,000$ days for middle term, and $T_{\mathrm{p}}<0.17$ days for short term (see Fig. 1). It will be found from the values of $m$ for each term that the hazard rate for long term is increasing, that for middle term is decreasing, and that for short term is nearly constant.

Once the values for $K$ and $m$ are obtained, we can calculate the probability density $f(t)$, and the cumulative probability $F(t)$ from Eqs. (1) and (2). They are expressed as functions of time $t$ measured from the appearance of precursors. In Fig. 3, the solid and dashed lines represent observed and calculated cumulative probability respectively. The density functions per day $f(t) \Delta t(\Delta t=1$ day) for each term are shown by dashed lines in Fig. 4.

Next, we calculate the conditional probability $P_{\mathrm{c}}(t)$ of earthquake occurrence within a short time interval $\tau$ under the condition that earthquake has not occurred until time $t$. We can write $P_{\mathrm{c}}(t)$ as

$$
P_{\mathrm{c}}(t)=\frac{R(t)-R(t+\tau)}{R(t)}
$$

The solid lines in Fig. 4 represent the conditional probability in the case of $\tau=1$ day. $P_{\mathrm{c}}(t)$ for short term is nearly constant because of $m \simeq 0$, while $P_{\mathrm{c}}(t)$ for long term increases monotonously, and that for middle term decreases monotonously corresponding to positive and negative $m$ respectively.

Here we introduce a parameter $r$ which is related to the reliability of each term of precursors. The reliability parameter $r$ is defined using previous data as

$$
r=\frac{N_{\mathrm{e}}}{N_{\mathrm{p}}} \text {, and } N_{\mathrm{p}}=N_{\mathrm{e}}+\bar{N}_{\mathrm{e}}
$$

where $N_{\mathrm{p}}$ is the total number of precursors (genuine and spurious) of each term, $N_{\mathrm{e}}$ is the number of genuine precursors which are followed by corresponding earthquakes 

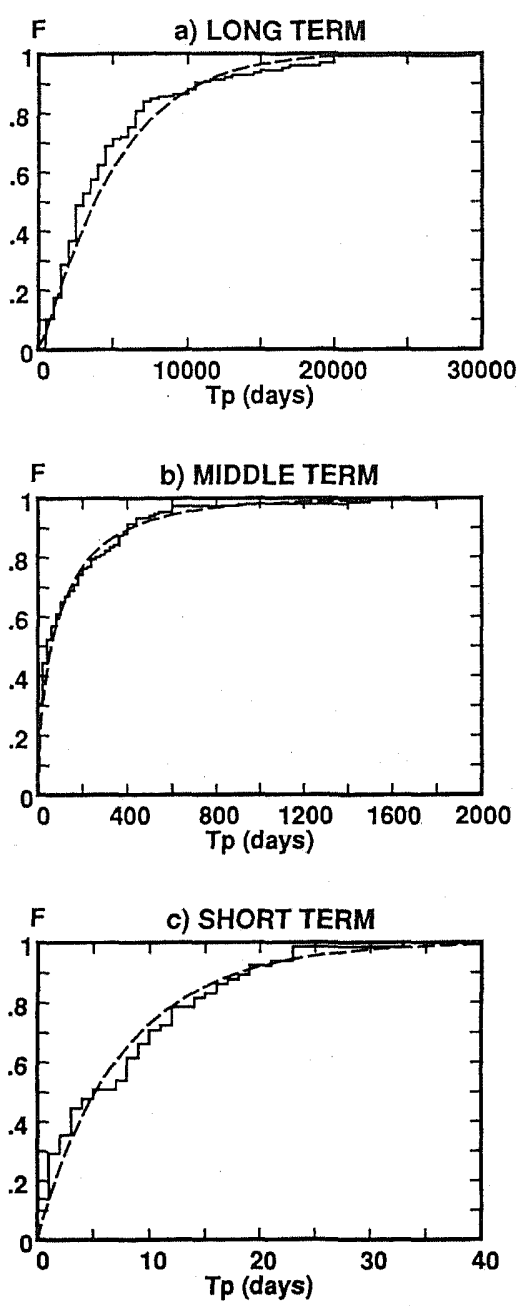

Fig. 3

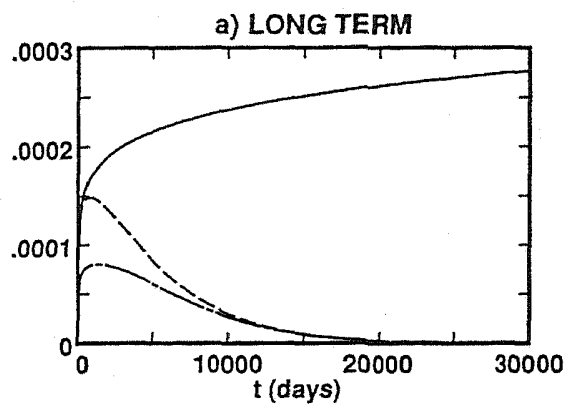

b) MIDDLE TERM

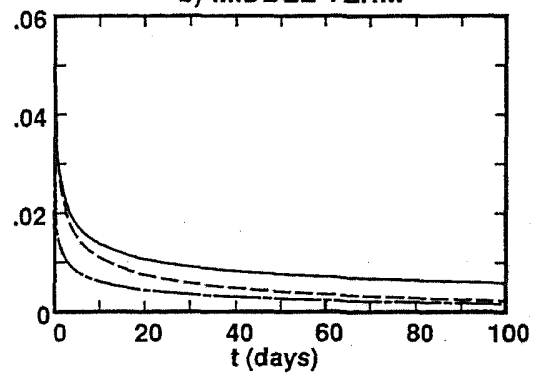

c) SHORT TERM

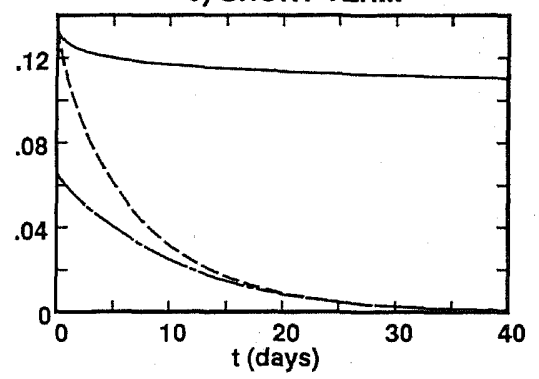

Fig. 4

Fig. 3. Observed (solid lines) and calculated (dashed lines) cumulative curves of precursor time $\left(T_{\mathrm{p}}\right)$ for each term. Calculated curves are obtained from Eq. (2) (see the text) by applying the values of $K$ and $m$ in Table 2.

Fig. 4. Probability density per day $f(t) \Delta t(\Delta t=1$ day), conditional probability $P_{\mathfrak{c}}(t)\left(\tau=1\right.$ day), and modified conditional probability $P_{\mathrm{cr}}(t)(\tau=1$ day, $r=0.5)$ are shown by dashed, solid, and chain lines, respectively.

and $\bar{N}_{\mathrm{e}}$ is the number of spurious precursors which are not followed by earthquakes. In this definition, $r$ is constant for each term. However, we cannot actually obtain $\bar{N}_{\mathrm{e}}$ from the data of S.V.R.D. (1990) because the data do not contain precursors which are not followed by corresponding earthquakes. Therefore, $r$ should be estimated using

Vol. 38 , No. 6, 1990 
other data set or basing on some assumptions when this method is applied to an actual case study.

According to the Bayes' theorem, the conditional probability will be modified into $P_{\mathrm{cr}}(t)$ as

$$
P_{\mathrm{cr}}(t)=\frac{r\{R(t)-R(t+\tau)\}}{r R(t)+(1-r)},
$$

using $r$ defined above. This expression is nearly the same as Eq. (7) in Vere-Jones (1978). Graphs for the modified conditional probability $P_{\mathrm{cr}}(t)$ for each term are displayed by chain lines in Fig. 4 in the case of $r=0.5$. It can be seen from Fig. 4 that as $t$ becomes large enough, $P_{\mathrm{cr}}(t)$ for every term decreases because of the effect of $r$.

Finally we combine the probability of three terms. According to Utsu $(1977,1983)$, the total probability $P(t)$ is given as

$$
P(t)=\frac{1}{1+\left(\frac{1}{p_{l}\left(t-t_{l}\right)}-1\right)\left(\frac{1}{p_{m}\left(t-t_{m}\right)}-1\right)\left(\frac{1}{p_{s}\left(t-t_{s}\right)}-1\right) /\left(\frac{1}{p_{0}}-1\right)^{2}},
$$

where $p_{t}(t), p_{m}(t)$, and $p_{s}(t)$ are conditional probability defined by Eq. (9) for long, middle and short term respectively. $t_{l}, t_{m}$, and $t_{s}$ are the appearance times of precursors for each term and $p_{0}$ is the average rate of earthquake occurrence per unit time, that is one day in our case. If $p_{i}(i=l, m, s)$ is less than $p_{0}, p_{i}$ is constrained to be equated to $p_{0}$. In special cases that $1 \gg p_{0}, 1 \gg p_{i}(i=l, m, s)$ and $1 \gg p_{0}\left(p_{l} / p_{0}\right)\left(p_{m} / p_{0}\right)\left(p_{s} / p_{0}\right)$, Eq. (10) is transformed into

$$
P(t)=p_{0} \cdot \frac{p_{l}\left(t-t_{l}\right)}{p_{0}} \cdot \frac{p_{m}\left(t-t_{m}\right)}{p_{0}} \cdot \frac{p_{s}\left(t-t_{s}\right)}{p_{0}},
$$

where $p_{i}(t) / p_{0}(i=l, m, s)$ is called probability gain of each term by Aki (1981) and is also called risk enhancement factor by Vere-Jones (1978) or predictive ratio by Kagan and Knopoff (1977).

Now we can calculate the time-varying total probability of occurrence per unit time by using Eq. (10) or (11) when precursors of three terms are observed under the condition that earthquake has not occurred till the current time.

\section{Application to the Izu-Oshima-Kinkai Earthquake of 1978}

The Izu-Oshima-Kinkai earthquake of $1978(M=7.0)$ is one of the well-known earthquakes in Japan which were preceded by many precursors. In order to apply the method described in the previous section, we need values of $T_{\mathrm{p}}$ and $r$ for long, middle and short terms which are denoted as $t_{l}, t_{m}, t_{s}, r_{l}, r_{m}$, and $r_{s}$, respectively, as well as the value of $p_{0}$. The list of precursors and $T_{\mathrm{p}}$ data of this earthquake which are compiled by S.V.R.D. (1990), are shown in Table 3 for only the disciplines classified as long, middle or short terms. The numbers of long, middle, and short term precursors are one, twelve, and two, respectively.

From the value listed in Table $3, t_{l}$ is equated to 4,784 days for the long term. The 
Table 3. $T_{\mathrm{p}}$ data of each precursor and their origins for Izu-Oshima-Kinkai earthquake (1978) which are compiled by S.V.R.D. (1990). Only disciplines classified as long, middle and short terms are listed.

\begin{tabular}{llcl}
\hline Term & \multicolumn{1}{c}{ Discipline } & $T_{\mathbf{p}}$ (day) & Reference \\
\hline \multirow{2}{*}{ Long } & \multicolumn{1}{c}{ Seismic pre-activity } & $\begin{array}{c}4,784^{*} \\
(4,78)^{* *}\end{array}$ & Suzuki (1985) \\
& & 65 & Honkura (1978) \\
\hline \multirow{2}{*}{ Middle } & Earth current & 200 & Yukutake et al. (1978) \\
& Resistivity & 90 & Honkura and Koyama (1978) \\
& Resistivity & 60 & Honkura and Koyama (1978) \\
& Resistivity & 180 & Geological Survey of Japan (1978) \\
& Radon & 90 & Geological Survey of Japan (1978) \\
& Radon & 30 & Geological Survey of Japan (1978) \\
& Radon & 14 & Geological Survey of Japan (1978) \\
& Radon & 10 & Geological Survey of Japan (1978) \\
& Radon & 90 & Wakita (1980) \\
& Radon & 5 & Wakita (1980) \\
& Radon & 40 & Takahashi and Tsuneishi (1978) \\
& Component of groundwater, gas & 1.8 & Tsumura et al. (1978) \\
& Foreshock & 0.7 & Suzuki (1985) \\
\hline \multirow{2}{*}{ Short } & Foreshock & &
\end{tabular}

* Corrected value by counting leap days. ${ }^{* *}$ Original one which is listed in S.V.R.D. (1990).

difference between two $T_{\mathrm{p}}$ data for the short term (foreshock) comes from the different definition of foreshock especially as to the lower limit of magnitudes. We take here the longer value, that is 1.8 days as $t_{s}$. Regarding the middle term, we propose a model that $t_{m}$ and $r_{m}$ have renewed values at every time when new precursors are observed in the time sequence. Assuming that the more precursors are observed, the larger $r_{m}$ becomes, we estimate $r_{m}$ as ${ }_{i} r_{m}$ which is defined as

$$
{ }_{i} r_{m}=1-\left(1-r_{m 0}\right)^{i} \text {, }
$$

where $i$ is time sequential number of middle term precursors and $r_{m 0}$ is a constant. As for the reliability parameters $r_{l}, r_{m 0}, r_{s}$, we do not have enough information to evaluate them except the estimation done by Utsu (1979). So, we will show probability changes for three sets of values of the parameters. The first set is that $r_{l}=0.5, r_{m 0}=0.01$, and $r_{s}=0.1$; the second set is that $r_{l}=0.5, r_{m 0}=0.1$, and $r_{s}=0.1$; and the last set is that $r_{l}=0.1, r_{m 0}=0.1$, and $r_{s}=0.5$. The results when other sets of values are taken can be estimated by the differences of the results for these three sets. Next, we estimate the average rate of earthquake occurrence per day as $1 /(365 \times 100)$ considering the average recurrence time of large earthquakes around the epicentral region.

On the basis of the above estimation, the total conditional probability of earthquake occurrence per day on condition no earthquake has occurred till the current time is calculated from Eq. (10). The probability change for each set of reliability parameters is shown in Figs. 5 to 7. The arrow in these Figures represents the occurrence time of the main shock which is the origin of time axis. Cross mark, empty and solid triangles

Vol. 38, No. 6,1990 


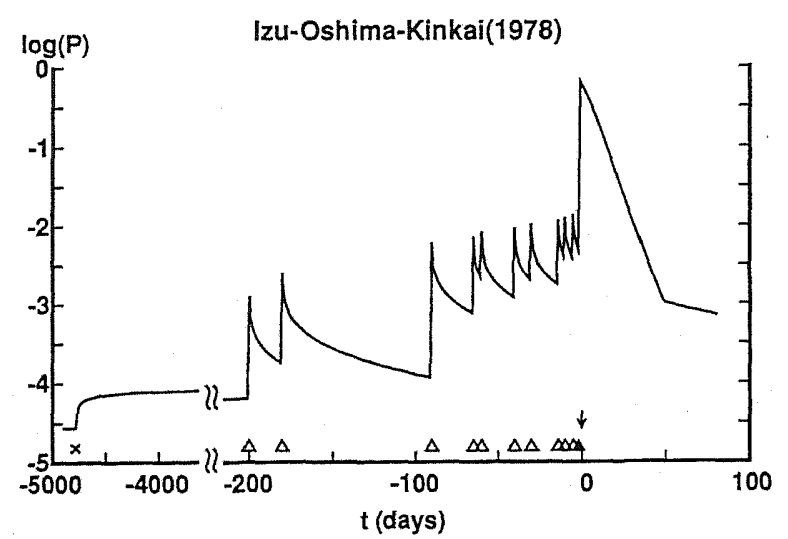

Fig. 5

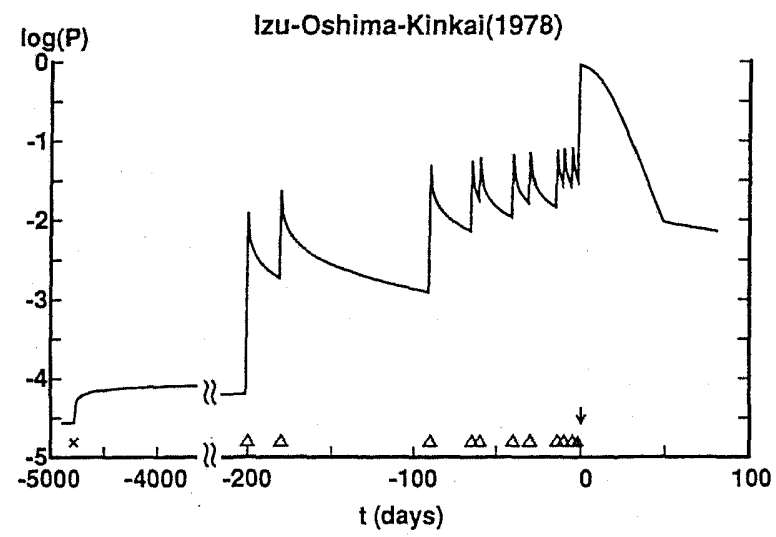

Fig. 6

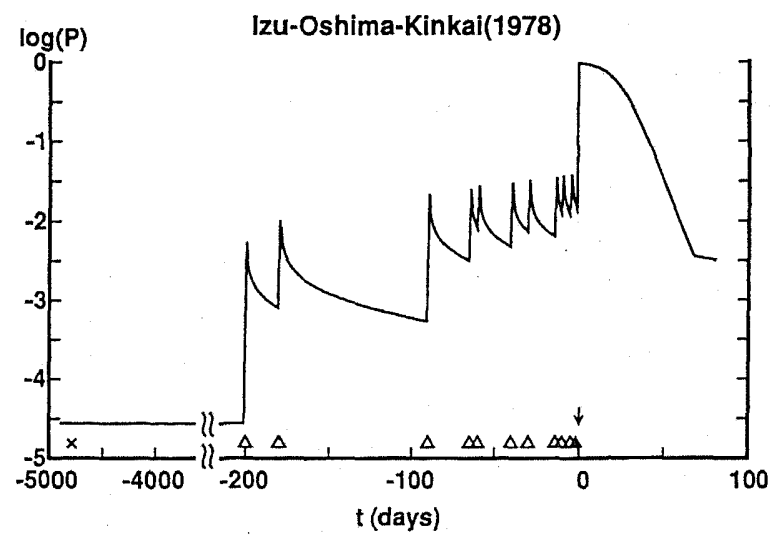

Fig. 7 
denote long, middle and short term precursors respectively. Although the absolute value of $P$ in each Figure differs from one another depending on the values of $r_{l}, r_{m 0}$, and $r_{s}$, the pattern of change of $P$ is not much different. The main factors which affect the pattern are the number of independent precursors (three in our case) and the values of $K, m$ for each term. The effect of the appearance of the long term precursor, that is seismic preactivity in this case, lasts almost constantly except a short period after its appearance. At every time when middle term precursor are observed, the probability steps up and decreases with time. The level of-stepping up increases corresponding to Eq. (12). Short term precursor, foreshock activity, raises probability again simultaneously with its appearance. After taking the maximum value, the probability decays with time until short term effect disappears at the rate mainly depending on $p_{s}$ 's decreasing rate. The main shock actually occurred at nearly the time of the maximum of the probability owing to the appearance of short term precursor, foreshock activity. However, it should be noted that we have not yet found an effective method to distinguish foreshock activity from others before the occurrence of main shock.

\section{Discussion}

The precursor data compiled by S.V.R.D. are not homogeneous in the meaning that the definitions of each precursor have much variety and there is little assurance that the observations of precursors were performed continuously. Nevertheless, the fact that the calculated lines shown in Fig. 2 fit the data fairly well may imply that the precursors really have such properties as a first approximation. At the same time, another interpretation could also be possible that some biases of observers produce such apparent properties. For example, there might be a possibility that the monotonous decrease of probability density for middle and short terms shown by dashed lines in Fig. 4 expresses merely the tendency of decrease of observers' attention. In order to clarify the fact, it is necessary to investigate the reliability of each precursor objectively by going back to the original data.

As mentioned in the previous section, the magnitude dependence of $T_{\mathbf{p}}$ may affect somewhat the results. According to the figures in S.V.R.D. (1990) representing the relation between magnitude and $T_{\mathrm{p}}$ for each discipline, there is a possibility that $T_{\mathrm{p}}$ depends on magnitude even in the magnitude range greater than or equal to 6.5 for the long term precursors. In order to evaluate the dependence on magnitude for the long term we divided the data into two data sets: one is the data set for main shock magnitude $6.5 \leqq M<7.5$, and the other for magnitude $7.5 \leqq M$. The values of parameters obtained

Fig. 5. Variation of total conditional probability for the Izu-Oshima-Kinkai earthquake of 1978. The arrow represents the occurrence time of the main shock which is the origin of the time axis. The appearance times of long, middle, and short term precursors listed in Table 3 are denoted by a cross mark, empty, and solid triangles respectively. $r_{l}=0.5, r_{m 0}=0.01$, and $r_{s}=0.1$ are assumed.

Fig. 6. Same as Fig. 5 but $r_{l}=0.5, r_{m 0}=0.1$, and $r_{s}=0.1$ are assumed,

Fig. 7. Same as Fig. 5 but $r_{l}=0.1, r_{m 0}=0.1$, and $r_{s}=0.5$ are assumed.

Vol. 38 , No. 6, 1990 
for the former data set are $K=3.6 \mathrm{E}-6, m=0.63$, and those for the latter data set are $K=1.7 \mathrm{E}-5, m=0.26$, respectively. Although the differences of values are not small, the property of increasing hazard rate does not change and the pattern of the probability change for the Izu-Oshima-Kinkai earthquake is not much affected.

In the present study, the regional differences are not considered because we do not have sufficient data to get statistically reliable results. However, it is expected that the parameters $K, m$, and $r$ have regionality on the analogy of recurrence time analysis made by Rikitake (1976).

When getting Eq. (10), we assumed that long, middle and short terms are independent mutually. Strictly speaking, however, they are not independent because they are considered to appear in the course of increase of stress in the focal region. If they are regarded as dependent on each other, the total probability becomes smaller than that given by Eq. (10). Rhoades (1989) proposed a different method which gives a smaller total probability. The essence of his method is based on an idea that the independent information will raise the reliability of precursors. However, if we want to take dependence between precursors into consideration, it is necessary to clarify physical mechanisms of appearance of each precursor.

\section{Conclusion}

(1) Disciplines of precursors are classified into three groups: long-middle and short terms-and a method is proposed to calculate the total conditional probability which varies with time and depends on reliability parameter for each term.

(2) By analyzing precursor time data using Weibull distribution function, it is found that the hazard rates for the long, middle, and short terms have increasing, decreasing, and nearly constant properties respectively.

(3) Introducing of reliability parameter $r(<1)$ has an effect to make the conditional probability of every term decrease monotonously long after the appearance of each precursor.

(4) In the case of the Izu-Oshima-Kinkai earthquake of 1978, the pattern of change of total conditional probability is simulated for three sets of values of reliability parameters.

We thank H. Ito, T. Yokota, T. Odaka, and M. Seino for valuable suggestions and appreciate all colleagues of S.V.R.D. of Meteorological Research Institute who willingly provided us with useful data. We are also specially grateful to two anonymous reviewers for their helpful comments.

\section{REFERENCES}

Aki, K., A probabilistic synthesis of precursory phenomena, in Earthquake Prediction-An International Review, ed. D. W. Simpson and P. G. Richards, pp. 566-574, A.G.U., Washington D.C., 1981.

E.C.S.S. (Earthquake Countermeasure Section of Shizuoka Prefectural Government), Present Status of the Analysis of Precursory Phenomena of Earthquakes, Shizuoka, 146 pp., 1985 (in 
Japanese).

E.C.S.S. (Earthquake Countermeasures Section of Shizuoka Prefectural Government), Whole Image of the Tonankai Earthquake-Reinvestigation in Shizuoka Prefecture, Shizuoka, 256 pp., 1986 (in Japanese).

Geological Survey of Japan, Change in radon concentration in ground water before and after the Izu-Oshima-Kinkai earthquake of 1978, Rep. Coord. Comm. Earthq. Predict., 20, 109-113, 1978 (in Japanese).

Hagiwara, Y., Probability of earthquake occurrence as obtained from a Weibull distribution analysis of crustal strain, Tectonophysics, 23, 313-318, 1974.

Hamada, K., Statistics of precursory phenomena to earthquakes occurring in and around Japan, Proceedings of Earthquake Prediction Research Symposium (1987), 243-249, 1987 (in Japanese).

Hamada, K., Classification of earthquake precursors and regularity of precursor appearances, Rep. Coord. Comm. Earthq. Predict., 39, 387-395, 1988 (in Japanese).

Honkura, Y., On a relation between anomalies in the geomagnetic and telluric fields observed at Nakaizu and the Izu-Oshima-Kinkai earthquake of 1978, Bull. Earthq. Res. Inst., Univ. Tokyo, 53, 931-937, 1978 (in Japanese).

Honkura, Y. and S. Koyama, Observation of short-period geomagnetic variations at Nakaizu (1), Bull. Earthq. Res. Inst., Univ. Tokyo, 53, 925-930, 1978 (in Japanese).

Kagan, Y. and L. Knopoff, Earthquake risk prediction as a stochastic process, Phys. Earth Planet. Inter., 14, 97-108, 1977.

Niazi, M., Regression analysis of reported earthquake precursors. I. Presentation of data, Pageoph, 122, 966-981, 1985.

Nishenko, S. P. and R. Buland, A generic recurrence interval distribution for earthquake forecasting, Bull. Seismol. Soc. Am., 77, 1382-1399, 1987.

Ogata, Y. and K. Katsura, Point-process models with linearly parameterized intensity for application to earthquake data, J. Appl. Probab., Special Volume 23a, 291-310, 1986.

Oike, K., Precursors and predictors of large earthquakes in China, Proceedings on the Chinese Earthquake Prediction by the 1977 Delegation of the Seismological Society of Japan, Seismological Society of Japan, Tokyo, 135-148, 1978 (in Japanese).

Rikitake, T., Recurrence of great earthquakes at subduction zones, Tectonophysics, 35, 335-362, 1976.

Rikitake, T., Earthquake Forecasting and Warning, Center for Academic Publications Japan Tokyo/D. Reidel Publishing Company, Tokyo, pp. 214-234, 1982.

Rikitake, T., Earthquake Precursors-Database for Earthquake Prediction, The University of Tokyo Press, Tokyo, 232 pp., 1986 (in Japanese).

Rikitake, T., Earthquake precursors in Japan: precursor time and detectability, Tectonophysics, 136, 265-282, 1987 a.

Rikitake, T., A practical approach to earthquake prediction basing on the empirical nature of precursors (2): Occurrence time, Zisin, Ser. 2, 40, 605-617, 1987 b (in Japanese).

Rhoades, D. A., Independence, precursors and earthquake hazard, Tectonophysics, 169, 199-206, 1989.

Scholz, C. H., L. R. Sykes, and Y. P. Aggarwal, Earthquake prediction: a physical basis, Science, 181, 803-810, 1973.

Suzuki, Y., Records on Foreshocks, Personal publication, 108-109, 1985 (in Japanese).

S.V.R.D. (Seismology and Volcanology Research Division), Database of earthquake precursors, 
Technical reports of the Meteorological Research Institute, Vol. 26, 329 pp., 1990 (in Japanese).

Sykes, L. R. and S. P. Nishenko, Probabilities of occurrence of large plate rupturing earthquakes for the San Andreas, San Jacinto and Imperial faults, California, 1983-2003, J. Geophys. Res., 89, 5905-5927, 1984.

Takahashi, $\mathrm{H}$, and $\mathrm{Y}$. Tsuneishi, Variations in chemical composition of thermal water before and after the 1978 Izu-Oshima-Kinkai earthquake, at Tokunaga-minami and Tsukigase in the Izu peninsula, Bull. Earthq. Res. Inst., Univ. Tokyo, 53, 987-994, 1978 (in Japanese).

Tsumura, K., I. Karakama, I. Ogino, and M. Takahashi, Seismic activities before and after the Izu-Oshima-Kinkai earthquake of 1978, Bull. Earthq. Res. Inst., Univ. Tokyo, 53, 675-706, 1978 (in Japanese).

Utsu, T., Probabilities in earthquake prediction, Zisin, Ser. 2, 30, 179-185, 1977 (in Japanese).

Utsu, T., Calculation of the probability of success of an earthquake prediction (in the case of Izu-Oshima-Kinkai earthquake of 1978), Rep. Coord. Comm. Earthq. Predict., 21, 164-166, 1979 (in Japanese).

Utsu, T., Probabilities associated with earthquake prediction and their relationships, Earthq. Predict. Res., 2, 105-114, 1983.

Utsu, T., Estimation of parameters for recurrence models of earthquakes, Bull. Earthq. Res. Inst., Univ. Tokyo, 59, 53-66, 1984.

Vere-Jones, D., Earthquake prediction-A statistician's view, J. Phys. Earth, 26, 129-146, 1978.

Wakita, H., Geochemical precursors of the 1978 Izu-Oshima-Kinkai earthquake, Proceedings of Earthquake Prediction Research Symposium (1980), 71-76, 1980 (in Japanese).

Yong, C., K. Tsoi, C. Feibi, G. Zhenhuan, Z. Qijia, and C. Zhangli, The Great Tangshan Earthquake of 1976-An Anatomy of Disaster, Pergamon Press, Oxford, pp. 96-144, 1988.

Yukutake, T., T. Yoshino, H. Utada, and T. Shimomura, Time variations observed in the earth resistivity on the Oshima volcano before the Izu-Oshima-Kinkai earthquake on January 14, 1978, Bull. Earthq. Res. Inst., Univ. Tokyo, 53, 961-972, 1978 (in Japanese). 